\title{
P04-2-52 Poster session
}

\section{Elucidation of PIEZO1 as Potential Therapeutic Target for Statin}

\author{
Tzyy Yue Wong ${ }^{1}$, Sheng-Nan Chang ${ }^{2,3}$, Chia-Ti Tsai ${ }^{4}$, Jou-Wei Lin ${ }^{3}$, Juey-Jen Hwang ${ }^{4}$, Pei- \\ Wen Cheng ${ }^{5}$
}

\begin{abstract}
${ }^{I}$ Department of Medical Education and Research, Kaohsiung Veterans General Hospital, Kaohsiung, Taiwan, ${ }^{2}$ National Taiwan University College of Medicine, Graduate Institute of Clinical Medicine, Taipei, Taiwan, ${ }^{3}$ Division of Cardiology, Department of Internal Medicine, National Taiwan University Hospital Yun-Lin Branch, Dou-Liu City, Taiwan, ${ }^{4}$ Division of Cardiology, Department of Internal Medicine, National Taiwan University Hospital, Taipei, Taiwan, ${ }^{5}$ Department of Education and Research, Kaohsiung Veterans General Hospital, Kaohsiung, Taiwan
\end{abstract}

\section{Background}

Cardiac cells contract and relax by arrangement of intracellular cytoskeletal protein such as actin, which is regulated through Rho/ROCK signaling pathway in muscle cells. The stretching force of contraction and relaxation can affect ion channels expressed on cardiac cells. PIEZO1 is a stretch-activated ion channel (SAC) first identified in Neuro2A cell line by Coste et al. in 2010. The transduction of calcium ions through SAC such as transient receptor potential cation channel subfamily V member 1 (TRPV1) was found to promote survival, and hypertrophy in muscle cells. Statin, the cardiac disease drug can inhibit Rho/ROCK signaling to activate endothelial-derived NOS (eNOS), leading to improved vascular function. We hypothesize that PIEZO1 plays a role in actin organization through Rho/ROCK pathway, and statin can regulate, or inhibit PIEZO1 to suppress Rho/ROCK.

Methods

We used cyclic stretch of $5 \%, 1 \mathrm{~Hz}$ to stimulate AC16 cardiac cells for $24 \mathrm{~h}$ to mimic in vivo heartbeat of cardiomyocytes, analyzed PIEZO1 expression and AC16 biomarker DESMIN by immunofluorescence assay. We analyzed calcium transduction using calcium green fluorescence probe. We verified whether Rho/ROCK is regulated by PIEZO1 using PIEZO1 inhibitor (GsMTx4). The statin was used to treat cells with PIEZO1 knockdown. We created 3D culture system for AC16 and then subject to stretching. The PIEZO1 expression was analyzed, and verified if PIEZO1 is correlated with actin organization in the $3 \mathrm{D}$ culture system with and without statin in order to create a model that can simulate an in vivo living tissue.

Results

After stretching the AC16 cells at 5\%,1Hz for 24h, PIEZO1 was found to be localized to perimeter of the cells compared to that control (No stretch) (Fig. 1A). Interestingly, the level of phosphor-AKT was decreased after the stretch. Currently, this is an ongoing project, and the function of PIEZO1 in the AC16 cells is being investigated.

Conclusions

PIEZO1 may play important role in actin organization of cardiac cells, wherein the mechanism may be related to statinmodulated Rho/ROCK pathway.

Keywords

PIEZO1, Rho/ROCK, cardiac disease 